\title{
Learning Biochemistry by Chocolate
}

\author{
Guedes,M.C.; Cayres,C.B.;Mercado,G.P.;Pimentel,E.F.;Silva,G.F. \\ Quimica-IMAPES
}

Both sensations and biochemical reactions taken place or promoted during ingestion of chocolate were the motivation for investigating the organic compounds present in this source. Cocoa and chocolate are composed by several substances, among them, aminoacids and alkaloids. The objective of this investigation was to purpose a contextured approach of biochemistry through the sensations and reactions involving aminoacids, theobromine and hormones. Methodology: 1. Theoretical part: constituted by theoretical and tutorial classes about aminoacids, theobromine and hormones involved at the metabolism; 2. Questionary: ten questions based upon theoretical classes, personal sensations and general aspects of chocolate; 3.Lecture: Cientific articles searched in periodics by own students as well as newspaper reports; 4. Experimental: Laboratory experiments including extraction, characterization, spectrometric quantification after specific reactions and identification by $\mathrm{Rf}$ comparison with standards on TLC from cocoa almonds and both powder cocoa and chocolate. The study was applied in 30 students from a chemistry college. Results: The results pointed out to a higher frequency of the students and to a increased interest from them by biochemistry issues and cientific lectures, as well as a satisfactory acquirement of theoretical and practice knowledge of aminoacids and hormones, spectrometry and chromatography.

Conclusion: A contextured approach is quite positive for learning biochemistry to chemists. 